DOI: $10.5277 /$ EPE120310

\title{
COMPREHENSIVE INTERPRETATION OF SAFETY OF WIDE WATER SUPPLY SYSTEMS
}

\begin{abstract}
A comprehensive method for safety assessment of water supply systems has been presented. The method includes both conditions of technical exploitation and quality of water delivered to consumers. In the analysis, the system capacity, appearance of failures on water mains as well as the chloroform concentration in transported water were taken into consideration. Four disjoint safety states have been defined (FS - full safety, ASM - acceptable safety menace, CSM - controlled safety menace, SL - safety loss) of water supply system. Moreover, the probabilities for the system to be in each of these states at the moment $t$ were derived based on the Markov processes.
\end{abstract}

\section{INTRODUCTION}

The main assignment of water supply system (WSS) is to deliver water of proper quality and required quantity to consumers. Water has to be delivered independently of interior or exterior conditions of WSS operation. These conditions are connected with numerous unforeseen events such as climate phenomena (flood, dry weather, hurricanes) and failures of technical equipments in water production or delivery subsystems. Continuous water supplying to inhabited territory, industrial and service areas secures proper development of administrative regions. Therefore water supply systems are regarded as a critical infrastructure which guaranties safety of city life [1-4].

Present water supply systems in developed countries do not contend with problem of water quantity but rather they have problems with its quality and its continuous delivery when random accidents happen. Contemporary circumstance of WSS operation causes the necessity of continuous control of technical system safety in the wide

*Silesian University of Technology, Faculty Of Energy And Environmental Engineering, Institute of Water and Wastewater Engineering, ul. Konarskiego 18, 44-100 Gliwice, Poland; e-mail: Izabela.Zimoch@polsl.pl

**Silesian University of Technology, Faculty of Applied Mathematics, Institute of Mathematics, ul. Kaszubska 23, 44-101 Gliwice, Poland; e-mail: Ewa.Lobos@polsl.pl 
sense. Simultaneously, in the WSS management process the lack of comprehensive tool for safety assessment is observed.

Such tool should be practicable in the total field of system exploitation, from water intake to water consumers. Hence it seems that the application of reliability and risk theory as well computer software is intended in order to build detailed research methodology and the method of WSS operation assessment [5-14]. This problem has significant practical meaning and also it is difficult and inspiring from the scientific point of view.

Big water companies have certificates in quality management consistent with international standard ISO 9001:2001 [15]. They treat the certificates as priority which secures the control of water intake, its treatment and distribution processes, which guarantees WSS safety. It is connected with the realization of main aim indicated by EU Water Framework Directive 2000/60/EU [1]. This directive brings principles of balanced development into effect, and its aim is, inter alia, the assurance of water delivery for people, agriculture and industry. Next, quality standards included in ISO 9001:2001 and 8th article of Drinking Water Directive 98/83/EC [16] impose necessity of risk analysis on water companies. The risk analysis should take into consideration numerous dangers for water company activity as well for consumers' health. The most effective way which guarantees safety of WSS operation is to put into practice control techniques and risk assessment, based on Water Safety Plans. Water Safety Plans are also recommended in 3rd edition of Guideline for Drinking Water Quality by WHO [3] as innovative ideas of identification, assessment, and management of risk and safety of WSS.

\section{TOPICAL ISSUES OF WIDE WATER SUPPLY SYSTEMS OPERATION}

Many exploited WSS are dated back to 19th century but these systems unceasingly are extended together with the development of water treatment and distribution techniques. Economic progress of urban and industry regions was usually accompanied by the exploration for new water resources. Consequently, present wide water systems exploit a few independent water intakes. This fact increases reliability of WSS operation if unexpected pollution appears in one of water intakes or technical failure occurs in a water treatment plant.

In such WSS, high water treatment technologies are applied. These technologies are based on techniques of advanced oxidation, ozonization, coagulation, rapid filtration, active carbon sorption, and disinfection, so they allow one to produce water of very high quality, which is usually better than one required by Polish regulation and international law. Simultaneously, the progress of analytic laboratory technologies in testing water quality and its composition allow one to identify diverse contamination (with high accuracy and precision) which are present even in minimum amount. It is 
worth to stress that knowledge of harmfulness effect of some substances contained in water (on human health) is continually extended. Moreover, epidemic transmitted by water are better identified, which is important in order to prevent them and their material and environment consequences as well as human diseases or deaths.

Results of such incidental contaminations of water resources may be serious, e.g. those in: Australia, 1992 [17], Milwaukee, 1993 [18], Walkenton, 2000 [19], Nowy Targ, 2003 [20]. Such events appear accidentally, being rather rare but their consequences are immeasurable.

Today one of the most important problems in water management is rational exploitation of wide water distribution networks which in many cases are characterized by capacity, higher than necessary. It follows from the fact that water pipes have been built for many years in order to deliver water to all inhabitants and other consumers (industry, public services, etc.). Therefore water distribution subsystems have oversized water pipes built from many diverse materials and this causes difficulties in the preservation of water quality during its transport to consumers. This water transport redundancy is an effect of water demand decrease and innovative saving water technologies that are put in practice by factories and industrial plants. Thus the phenomena of water velocity decrease in water pipes (less than $0.1 \mathrm{~m} / \mathrm{s}$ ) and elongated time of water transport (even a few days) are observed. This causes the increasing risk of secondary water contamination because water distribution network becomes the chemical and bacteriological reactor. Lack of chemical stability intensifies corrosion processes in water pipes which next implies sediments accumulation. The sediments are basis for reproduction of microorganisms, creating colonies in the form of biofilm; thus water is also biologically instable.

Water distribution networks as technical systems are characterized by failure occurrence of different water infrastructure elements. The frequency of these failures is determined by age and material of water-pipes. Lack of water in failure area causes significant changes in operation conditions of remaining water pipes in working order. Changes in the direction of water flow and repair works increased risk of secondary contamination. Also higher costs of water delivery which arise during failure (ballasted the water company as well consumers) are consequences of these events.

Since exploitation of WSS involves occurrence of random events which may cause public health menace as well the increase of system work costs, it is necessary to constantly monitor and analyze the risk within the confines of Water Safety Plans [3]. The procedures of these plans assume precise safety analysis of WSS operation based on probability calculus and reliability theory, which should result in measurable risk assessment. Thus safety and risk are inseparable concepts in theory of WSS exploitation. The safety reliability is expressed in terms of probability that catastrophic results will not appear whereas the risk is defined as the product of the probability (or frequency) and results of undesirable events. The results are usually measured by means of costs, the number of hospitalized water consumers, environmental losses, etc. 
The necessity of so extensive analyses requires a comprehensive approach to the problem of assessment of WSS safety. The method of this assessment should also include the interpretation of possible exploitation states of WSS, expressed by transported water quality, failures of technological elements of water treatment arrangements as well water pipe networks.

\section{METHOD OF COMPREHENSIVE ANALYSIS OF WSS SAFETY}

The method presented below takes into consideration only wide water supply systems with at least two independent water intakes, such that the production significantly exceeds average daily water demand (i.e. the considered systems have production redundancy). An important assumption is that single failure in water production subsystem not necessarily leads to full loss of possibility of water delivery to consumers, safe for their health. The assessment of WSS safety depends on the following three criteria: the quality of transported water, water production capacity, and the nonfailure work of main water pipes [21].

In the study, we have defined three quality states depending on the greatest concentration of chloroform in transported water in all monitored points of distribution system (under the assumption of correct water production subsystem work, i.e. the concentration of chloroform in treated water is lower than $2-3 \mu \mathrm{g} / \mathrm{dm}^{3}$ ):

- first quality state - the concentration of $\mathrm{CHCl}_{3}$ is lower than $30 \mu \mathrm{g} / \mathrm{dm}^{3}$ (very probable exploitation event),

- second quality state - the concentration of $\mathrm{CHCl}_{3}$ is higher than or equal to $30 \mu \mathrm{g} / \mathrm{dm}^{3}$ and lower than $90 \mu \mathrm{g} / \mathrm{dm}^{3}$ (probable exploitation event),

- third quality state - the concentration of $\mathrm{CHCl}_{3}$ is higher than or equal to $90 \mu \mathrm{g} / \mathrm{dm}^{3}$ (not impossible exploitation event).

The quality state at which the system stays is dependent, to some extent, on exploitation conditions such as the rate of water production, water demand, the complexity of water infrastructure, etc. Thus in the comprehensive safety analysis it is intentional to take into consideration two states of water distribution subsystem (WDS) efficiency and four states of production capacity. States of WDS efficiency in the research are identified with failures occurring in water main - in the first state there are no failures in WDS and in the second state a failure in WDS occurs. Production capacity, which is related to the number of water treatment plants operating in WSS, is commonly [22] classified into four states:

- first state (the full efficiency of production) - production capacity is equal to water demand by consumers: $Q=Q_{n}$,

- second state (the partial efficiency of production) - the production capacity is decreased to $70 \%$ of water demand as a result of short duration break down of WPS elements: $0.7 Q_{n} \leq Q<Q_{n}$, 
- third state (the controlled hazard of efficiency of production) - the production capacity is between $30 \%$ and $70 \%$ of water demand which is caused by serious failures of WPS elements: $0.3 Q_{n} \leq Q<0.7 Q_{n}$,

- fourth state (unreliability of efficiency of production) - the production capacity achieves at most $30 \%$ of water demand because of diverse random events: $Q<0.3 Q_{n}$.

Based on states listed above, we define four states of safety of WSS: full safety (FS), acceptable safety menace (ASM), controlled safety menace (CSM), and safety loss (SL) [21]. The precise classification of above safety sates is given in Table 1 . Such defined safety states make possible to specify action procedures that result from WSP as well to assess the risk of both consumers and producers.

Table 1

Safety states

\begin{tabular}{|l|l|c|c|c|}
\hline \multicolumn{2}{|c|}{ State } & $\mathrm{CHCl}_{3} \in\langle 0,30)$ & $\mathrm{CHCl}_{3} \in\langle 30,90)$ & $\mathrm{CHCl}_{3} \geq 90$ \\
\hline \multirow{4}{*}{$\begin{array}{l}\text { No failure } \\
\text { in WDS }\end{array}$} & $Q=Q_{n}$ & FS & ASM & SL \\
\cline { 2 - 5 } & $0.7 Q_{n} \leq Q<Q_{n}$ & FS & CSM & SL \\
\cline { 2 - 5 } & $0.3 Q_{n} \leq Q<0.7 Q_{n}$ & ASM & CSM & SL \\
\cline { 2 - 5 } & $Q<0.3 Q_{n}$ & CSM & CSM & SL \\
\hline \multirow{4}{*}{$\begin{array}{l}\text { A failure } \\
\text { in WDS }\end{array}$} & $Q=Q_{n}$ & ASM & CSM & SL \\
\cline { 2 - 5 } & $0.7 Q_{n} \leq Q<Q_{n}$ & ASM & CSM & SL \\
\cline { 2 - 5 } & $0.3 Q_{n} \leq Q<0.7 Q_{n}$ & CSM & SL & SL \\
\cline { 2 - 5 } & $Q<0.3 Q_{n}$ & SL & SL & SL \\
\hline
\end{tabular}

$Q_{n}$ - water demand in $\mathrm{m}^{3} /$ day, concentration of $\mathrm{CHCl}_{3}$ in $\mu \mathrm{g} / \mathrm{dm}^{3}$.

According to legal acts, if the chloroform concentration exceeds triple maximum permissible concentration, then such states are classified as safety loss because the public health hazard appears. States of controlled safety menace require the increase of check-up of water treatment effects and monitoring frequency of transported water quality (concentration of chloroform). Such actions are connected with costs but they guarantee the safety of public health as well as WSS management.

Fundamental assumption of presented model is that the system has at least two water treatment plants and the system has possibility of production at least $70 \%$ of water demand in spite of single failure in water main. Then it is possible to supply almost $100 \%$ of water demand by consumers because there is stored water in the system (in tanks and in water pipes). Thus cases where the water main is damaged but the system produces at least $70 \%$ of water demand and the concentration of chloroform is lower than $30 \mu \mathrm{g} / \mathrm{dm}^{3}$ (maximum acceptable level), are defined as acceptable safety menace. If concentration of chloroform does not exceed maximum acceptable level 
but water capacity of the system is between $30 \%$ and $70 \%$ of required water quantity, and additionally a failure in WDS is observed, then hydraulic parameters of distribution subsystem operation are significantly changed, for example the velocity of water flow in pipes is less which is conducive to the formation of chloroform and other disinfection by products as well secondary contamination of water. Thus such exploitation case requires the increase of monitoring intensity of chloroform in critical points of the system and it was classified as the state of controlled safety menace.

\section{PROBABILITIES OF DEFINED WSS SAFETY STATES}

Let us denote the probabilities that system is in described states related to water capacity of water production subsystem by $\alpha_{i}, i=1,2,3,4$, to WDS operation by $\beta_{i}$, $i=1,2$, and to transported water quality by $\gamma_{i}, i=1,2,3$. Definitions of listed probabilities are as follows:

$$
\begin{aligned}
& \alpha_{1}(t)=\operatorname{Pr}\left\{Q=Q_{n} \text { at time } t\right\}, \\
& \alpha_{2}(t)=\operatorname{Pr}\left\{0.7 Q_{n} \leq Q<Q_{n} \text { at time } t\right\}, \\
& \alpha_{3}(t)=\operatorname{Pr}\left\{0.3 Q_{n} \leq Q<0.7 Q_{n} \text { at time } t\right\}, \\
& \alpha_{4}(t)=\operatorname{Pr}\left\{Q<0.3 Q_{n} \text { at time } t\right\}, \\
& \beta_{1}(t)=\operatorname{Pr}\{W D S=1 \text { at time } t\}, \\
& \beta_{2}(t)=\operatorname{Pr}\{W D S=0 \text { at time } t\}, \\
& \gamma_{1}(t)=\operatorname{Pr}\left\{\mathrm{CHCl}_{3}<30 \text { at time } t\right\}, \\
& \gamma_{2}(t)=\operatorname{Pr}\left\{30 \leq \mathrm{CHCl}_{3}<90 \text { at time } t\right\}, \\
& \gamma_{3}(t)=\operatorname{Pr}\left\{\mathrm{CHCl}_{3} \geq 90 \text { at time } t\right\} .
\end{aligned}
$$

For simplicity, the independence of considered random variables (i.e. water capacity $Q$, water quality $\mathrm{CHCl}_{3}$, and water main operation WDS) is assumed. Then the probabilities that the system is in selected safety state at time $t$ may be calculated as

$$
\begin{gathered}
P_{F S}(t)=\beta_{1}(t)\left[\alpha_{1}(t)+\alpha_{2}(t)\right] \gamma_{1}(t) \\
P_{A S M}(t)=\beta_{1}(t)\left[\alpha_{3}(t) \gamma_{1}(t)+\alpha_{1}(t) \gamma_{2}(t)\right]+\beta_{2}(t)\left[\alpha_{1}(t)+\alpha_{2}(t)\right] \gamma_{1}(t) \\
P_{C S M}(t)=\beta_{1}(t)\left[\alpha_{4}(t) \gamma_{1}(t)+\left(\alpha_{2}(t)+\alpha_{3}(t)+\alpha_{4}(t)\right) \gamma_{2}(t)\right] \\
+\beta_{2}(t)\left[\left(\alpha_{1}(t)+\alpha_{2}(t)\right) \gamma_{2}(t)+\alpha_{3}(t) \gamma_{1}(t)\right] \\
P_{S L}(t)=\gamma_{3}(t)+\beta_{2}(t)\left[\alpha_{4}(t) \gamma_{1}(t)+\left(\alpha_{3}(t)+\alpha_{4}(t)\right) \gamma_{2}(t)\right]
\end{gathered}
$$


Each of considered processes of the change of state may be modeled by a stationary Markov process, i.e. the probability that the system is at the moment $t+\Delta t$ in the $j$ th state whereas it was in the $i$ th state at the moment $t$ equals

$$
P_{i j}(t+\Delta t)=\lambda_{i j} \Delta t+o(\Delta t)
$$

where $\lambda_{i j}$ is the transition intensity and $\lim _{\Delta t \rightarrow 0} \frac{o(\Delta t)}{\Delta t}=0$.

The simplest case is the case of WDS operation where we have only two states. The system is in state 1 (no failure) at $t+\Delta t$ if and only if at the moment $t$ it was in state 1 and during $\Delta t$ the state was unchanged or it was at the state 2 (failure) and during $\Delta t$ there was a change of state from 2 to 1 . Hence

$$
\begin{aligned}
\beta_{1}^{\prime}(t) & =\lim _{\Delta t \rightarrow 0} \frac{\beta_{1}(t+\Delta t)-\beta_{1}(t)}{\Delta t} \\
& =\lim _{\Delta t \rightarrow 0} \frac{\beta_{1}(t)\left[1-\lambda_{12}^{(\beta)} \Delta t-o(\Delta t)\right]+\beta_{2}(t)\left[\lambda_{21}^{(\beta)} \Delta t+o(\Delta t)\right]-\beta_{1}(t)}{\Delta t} \\
& =-\lambda_{12}^{(\beta)} \beta_{1}(t)+\lambda_{21}^{(\beta)} \beta_{2}(t) .
\end{aligned}
$$

Analogously

$$
\begin{aligned}
\beta_{2}^{\prime}(t) & =\lim _{\Delta t \rightarrow 0} \frac{\beta_{2}(t+\Delta t)-\beta_{2}(t)}{\Delta t} \\
& =\lim _{\Delta t \rightarrow 0} \frac{\beta_{1}(t)\left[\lambda_{12}^{(\beta)} \Delta t+o(\Delta t)\right]+\beta_{2}(t)\left[1-\lambda_{21}^{(\beta)} \Delta t-o(\Delta t)\right]-\beta_{2}(t)}{\Delta t} \\
& =\lambda_{12}^{(\beta)} \beta_{1}(t)-\lambda_{21}^{(\beta)} \beta_{2}(t) .
\end{aligned}
$$

The homogeneous system of linear differential equations

$$
\left\{\begin{array}{l}
\beta_{1}^{\prime}(t)=-\lambda_{12}^{(\beta)} \beta_{1}(t)+\lambda_{21}^{(\beta)} \beta_{2}(t) \\
\beta_{2}^{\prime}(t)=\lambda_{12}^{(\beta)} \beta_{1}(t)-\lambda_{21}^{(\beta)} \beta_{2}(t)
\end{array}\right.
$$

has a general solution:

$$
\left[\begin{array}{l}
\beta_{1}(t) \\
\beta_{2}(t)
\end{array}\right]=C_{1}\left[\begin{array}{l}
\lambda_{21}^{(\beta)} \\
\lambda_{12}^{(\beta)}
\end{array}\right]+C_{2} \exp \left[-\left(\lambda_{12}^{(\beta)}+\lambda_{21}^{(\beta)}\right) t\right]\left[\begin{array}{r}
1 \\
-1
\end{array}\right]
$$

Next, for three quality states we have the following formulas 


$$
\begin{aligned}
& \gamma_{1}^{\prime}(t)=\lim _{\Delta t \rightarrow 0} \frac{\gamma_{1}(t+\Delta t)-\gamma_{1}(t)}{\Delta t}= \\
& =\lim _{\Delta t \rightarrow 0}\left(\frac{\gamma_{1}(t)\left[1-\lambda_{12}^{(\gamma)} \Delta t-\lambda_{13}^{(\gamma)} \Delta t-o(\Delta t)\right]}{\Delta t}\right. \\
& \left.+\frac{\gamma_{2}(t)\left[\lambda_{21}^{(\gamma)} \Delta t+o(\Delta t)\right]+\gamma_{3}(t)\left[\lambda_{31}^{(\gamma)} \Delta t+o(\Delta t)\right]-\gamma_{1}(t)}{\Delta t}\right) \\
& =-\left(\lambda_{12}^{(\gamma)}+\lambda_{13}^{(\gamma)}\right) \gamma_{1}(t)+\lambda_{21}^{(\gamma)} \gamma_{2}(t)+\lambda_{31}^{(\gamma)} \gamma_{3}(t) \\
& \gamma_{2}^{\prime}(t)=\lim _{\Delta t \rightarrow 0} \frac{\gamma_{2}(t+\Delta t)-\gamma_{2}(t)}{\Delta t}= \\
& =\lim _{\Delta t \rightarrow 0}\left(\frac{\gamma_{1}(t)\left[\lambda_{12}^{(\gamma)} \Delta t+o(\Delta t)\right]+\gamma_{2}(t)\left[1-\lambda_{21}^{(\gamma)} \Delta t-\lambda_{23}^{(\gamma)} \Delta t-o(\Delta t)\right]}{\Delta t}\right. \\
& \left.+\frac{\gamma_{3}(t)\left[\lambda_{32}^{(\gamma)} \Delta t+o(\Delta t)\right]-\gamma_{2}(t)}{\Delta t}\right) \\
& =\lambda_{12}^{(\gamma)} \gamma_{1}(t)-\left(\lambda_{21}^{(\gamma)}+\lambda_{23}^{(\gamma)}\right) \gamma_{2}(t)+\lambda_{32}^{(\gamma)} \gamma_{3}(t) \\
& \gamma_{3}^{\prime}(t)=\lim _{\Delta t \rightarrow 0} \frac{\gamma_{3}(t+\Delta t)-\gamma_{3}(t)}{\Delta t}= \\
& =\lim _{\Delta t \rightarrow 0}\left(\frac{\gamma_{1}(t)\left[\lambda_{13}^{(\gamma)} \Delta t+o(\Delta t)\right]+\gamma_{2}(t)\left[\lambda_{23}^{(\gamma)} \Delta t+o(\Delta t)\right]}{\Delta t}\right. \\
& \left.+\frac{\gamma_{3}(t)\left[1-\lambda_{31}^{(\gamma)} \Delta t-\lambda_{32}^{(\gamma)} \Delta t-o(\Delta t)\right]-\gamma_{3}(t)}{\Delta t}\right) \\
& =\lambda_{13}^{(\gamma)} \gamma_{1}(t)+\lambda_{23}^{(\gamma)} \gamma_{2}(t)-\left(\lambda_{31}^{(\gamma)}+\lambda_{32}^{(\gamma)}\right) \gamma_{3}(t) \text {. }
\end{aligned}
$$

The matrix of system

$$
\left\{\begin{array}{l}
\gamma_{1}^{\prime}(t)=-\left(\lambda_{12}^{(\gamma)}+\lambda_{13}^{(\gamma)}\right) \gamma_{1}(t)+\lambda_{21}^{(\gamma)} \gamma_{2}(t)+\lambda_{31}^{(\gamma)} \gamma_{3}(t) \\
\gamma_{2}^{\prime}(t)=\lambda_{12}^{(\gamma)} \gamma_{1}(t)-\left(\lambda_{21}^{(\gamma)}+\lambda_{23}^{(\gamma)}\right) \gamma_{2}(t)+\lambda_{32}^{(\gamma)} \gamma_{3}(t) \\
\gamma_{3}^{\prime}(t)=\lambda_{13}^{(\gamma)} \gamma_{1}(t)+\lambda_{23}^{(\gamma)} \gamma_{2}(t)-\left(\lambda_{31}^{(\gamma)}+\lambda_{32}^{(\gamma)}\right) \gamma_{3}(t)
\end{array}\right.
$$

has three eigenvalues

$$
\begin{aligned}
& s_{0}=0, \quad s_{1}=\frac{1}{2}\left[-\left(\lambda_{12}^{(\gamma)}+\lambda_{13}^{(\gamma)}+\lambda_{21}^{(\gamma)}+\lambda_{23}^{(\gamma)}+\lambda_{31}^{(\gamma)}+\lambda_{32}^{(\gamma)}\right)+\sqrt{\Delta}\right] \\
& s_{2}=\frac{1}{2}\left[-\left(\lambda_{12}^{(\gamma)}+\lambda_{13}^{(\gamma)}+\lambda_{21}^{(\gamma)}+\lambda_{23}^{(\gamma)}+\lambda_{31}^{(\gamma)}+\lambda_{32}^{(\gamma)}\right)-\sqrt{\Delta}\right]
\end{aligned}
$$


where

$$
\begin{aligned}
\Delta= & \left(\lambda_{12}^{(\gamma)}+\lambda_{13}^{(\gamma)}+\lambda_{21}^{(\gamma)}+\lambda_{23}^{(\gamma)}+\lambda_{31}^{(\gamma)}+\lambda_{32}^{(\gamma)}\right)^{2} \\
& -4\left[\left(\lambda_{12}^{(\gamma)}+\lambda_{13}^{(\gamma)}+\lambda_{21}^{(\gamma)}\right)\left(\lambda_{23}^{(\gamma)}+\lambda_{31}^{(\gamma)}+\lambda_{32}^{(\gamma)}\right)+\left(\lambda_{21}^{(\gamma)}-\lambda_{31}^{(\gamma)}\right)\left(\lambda_{13}^{(\gamma)}-\lambda_{23}^{(\gamma)}\right)\right]
\end{aligned}
$$

Thus the general solution of system (7) is

$$
\begin{aligned}
& {\left[\begin{array}{l}
\gamma_{1}(t) \\
\gamma_{2}(t) \\
\gamma_{3}(t)
\end{array}\right]=} C_{0}\left[\begin{array}{l}
\lambda_{21}^{(\gamma)} \lambda_{31}^{(\gamma)}+\lambda_{23}^{(\gamma)} \lambda_{31}^{(\gamma)}+\lambda_{21}^{(\gamma)} \lambda_{32}^{(\gamma)} \\
\lambda_{12}^{(\gamma)} \lambda_{32}^{(\gamma)}+\lambda_{13}^{(\gamma)} \lambda_{32}^{(\gamma)}+\lambda_{12}^{(\gamma)} \lambda_{31}^{(\gamma)} \\
\lambda_{12}^{(\gamma)} \lambda_{23}^{(\gamma)}+\lambda_{13}^{(\gamma)} \lambda_{21}^{(\gamma)}+\lambda_{13}^{(\gamma)} \lambda_{23}^{(\gamma)}
\end{array}\right] \\
&+C_{1} \exp \left(-s_{1} t\right)\left[\begin{array}{l}
\lambda_{31}^{(\gamma)}-\lambda_{21}^{(\gamma)} \\
-\lambda_{12}^{(\gamma)}-\lambda_{13}^{(\gamma)}-\lambda_{31}^{(\gamma)}-s_{1} \\
\lambda_{12}^{(\gamma)}+\lambda_{13}^{(\gamma)}+\lambda_{21}^{(\gamma)}+s_{1}
\end{array}\right] \\
&+C_{2} \exp \left(-s_{2} t\right)\left[\begin{array}{l}
\lambda_{31}^{(\gamma)}-\lambda_{21}^{(\gamma)} \\
-\lambda_{12}^{(\gamma)}-\lambda_{13}^{(\gamma)}-\lambda_{31}^{(\gamma)}-s_{2} \\
\lambda_{12}^{(\gamma)}+\lambda_{13}^{(\gamma)}+\lambda_{21}^{(\gamma)}+s_{2}
\end{array}\right]
\end{aligned}
$$

Similarly, the probabilities of sates of WSS capacity at any time $t$ are described by the following homogeneous system of linear differential equations:

$$
\left\{\begin{array}{l}
\alpha_{1}^{\prime}(t)=-\left(\lambda_{12}^{(\alpha)}+\lambda_{13}^{(\alpha)}+\lambda_{14}^{(\alpha)}\right) \alpha_{1}(t)+\lambda_{21}^{(\alpha)} \alpha_{2}(t)+\lambda_{31}^{(\alpha)} \alpha_{3}(t)+\lambda_{41}^{(\alpha)} \alpha_{4}(t) \\
\alpha_{2}^{\prime}(t)=\lambda_{12}^{(\alpha)} \alpha_{1}(t)-\left(\lambda_{21}^{(\alpha)}+\lambda_{23}^{(\alpha)}+\lambda_{24}^{(\alpha)}\right) \alpha_{2}(t)+\lambda_{32}^{(\alpha)} \alpha_{3}(t)+\lambda_{42}^{(\alpha)} \alpha_{4}(t) \\
\alpha_{3}^{\prime}(t)=\lambda_{13}^{(\alpha)} \alpha_{1}(t)+\lambda_{23}^{(\alpha)} \alpha_{2}(t)-\left(\lambda_{31}^{(\alpha)}+\lambda_{32}^{(\alpha)}+\lambda_{34}^{(\alpha)}\right) \alpha_{3}(t)+\lambda_{43}^{(\alpha)} \alpha_{4}(t) \\
\left.\alpha_{4}^{\prime} t\right)=\lambda_{14}^{(\alpha)} \alpha_{1}(t)+\lambda_{24}^{(\alpha)} \alpha_{2}(t)+\lambda_{34}^{(\alpha)} \alpha_{3}(t)-\left(\lambda_{41}^{(\alpha)}+\lambda_{42}^{(\alpha)}+\lambda_{43}^{(\alpha)}\right) \alpha_{4}(t) .
\end{array}\right.
$$

The matrix of system (9) has four eigenvalues $s_{i}, i=0,1,2,3$ which should be calculated using numerical methods (one of them $s_{0}=0$ is obvious). If $\mathbf{v}_{i}, i=0,1,2,3$, are corresponding eigenvectors, then the general solution of (9) is given by

$$
\left[\begin{array}{l}
\alpha_{1}(t) \\
\alpha_{2}(t) \\
\alpha_{3}(t) \\
\alpha_{4}(t)
\end{array}\right]=C_{0} \mathbf{v}_{0}+C_{1} \exp \left(-s_{1} t\right) \mathbf{v}_{1}+C_{2} \exp \left(-s_{2} t\right) \mathbf{v}_{2}+C_{3} \exp \left(-s_{3} t\right) \mathbf{v}_{3}
$$




\section{CONCLUSION}

The proposed method of safety analysis is comprehensive and it takes into consideration various exploitation conditions of WSS operation. Namely, it includes daily water capacity of production subsystem, failures of water mains as well as changes of water quality in a distribution subsystem.

Such expressed problem of definition of four safety states (Table 1), including technical conditions of WSS operation and quality of transported water, was not considered yet.

Probabilities (1)-(4) that the system is at any moment $t$ in one of defined safety states can be calculated using formulas (6), (8), (10). For the last three probabilities suitable transition intensities are essential. Transition intensities of course change in time because WSS are dynamic technical systems whose exploitation conditions depend on random events. Hence it is necessary to collect complete data concerning failures on water main, changes of water quality in the system, and changes of water production level in order to actualize (in given fixed periods) appropriate transition intensities. In most of wide WSS such data bases are systematically collected what follows from existing management procedures and cost optimization. Although there are known examples of applications of Markov processes in safety assessment of technical systems [23-25], their usage requires large data bases covering information concerning the history of changes of safety states, which are difficult to build because of lack of needed specific data and they are time consuming (long period of observation is necessary).

Presented method may be applied to large water supply systems which are characterized by redundancy of economic capacity and big quantities of water stored in distribution subsystem (not only in storage tanks but also in water pipes). Such properties of the system guarantee its correct work with high probability in spite of single shortlived main water failure.

\section{ACKNOWLEDGEMENTS}

Scientific work was financed from the measures of National Center of Research and Development as a development research project No. N R14 0006 10: Development of comprehensive methodology for the assessment of the reliability and safety of water supply to consumers in the years 2010-2013.

\section{REFERENCES}

[1] Directive 2000/60/EC of the European Parliament and of the Council of 23 October 2000 establishing a framework for the Community action in the field of water policy, Official Journal, 2000, L 327, 1-73.

[2] Regulation of the Minister of Health, 2010.04.20 amending the regulation on the quality of water allowed for human consumption, Acts. 72nd item. 466 (in Polish).

[3] Guidelines for Drinking Water Quality, Vol. 1, 3rd Ed., WHO, Geneva 2004. 
[4] Water Safety Plans - Managing Drinking Water Quality from Catchment to Consumers, WHO, Geneva 2005.

[5] Haimes Y.Y., Risk Modeling, Assessment and Management, Wiley, New York, 1998.

[6] RAK J., TCHÓRZEWSKA-CIEŚlAK B., Five-parametric matrix to estimate risk connected with water supply system operation, Env. Prot. Eng., 2006, 32 (2), 37-46.

[7] GALE P., Using risk assessment to identify future research requirements, J. AWWA, 2002, 94 (9), $30-38$.

[8] Van Lieverloo H.M., Medema G., Van Der Kooij D., Risk assessment and risk management of faecal contamination in drinking water distributed without a disinfectant residual, J. Water SRT - Aqua, 2006, 55 (1), 25-31.

[9] Kato, S., Suzuki, M., YoKoI, H.And Yoda M., Japan's trial introduction of HACCP into water quality management, Water21, 2006, 8 (6), 39-40.

[10] ZІмосн I., Reliability and risk analysis usage for water supply system management, Polish J. Environ. St., 2008, 17 (3A), 622-626.

[11] HubBard D.W., The Failure of Risk Management, Wiley, New York, 2009.

[12] RAK J., Selected problems of water supply safety, Env. Prot. Eng., 2009, 35 (2), 23-28.

[13] KowALSKI D., Water quality management in a water supply system, Ochrona Środowiska, 2009, 31(3), 37-40 (in Polish).

[14] AVEN T., A conceptual framework for risk assessment and risk management, J. Polish Safety Reliab. Assoc., 2010, 1, 15-27.

[15] ISO 9001:2001 Quality Management.

[16] Drinking Water Directive 98/83/EC, Council Directive of 3 November 1998 on the quality of water intended for human consumption.

[17] KABZIŃSKI A., KABZIŃSKI T., Toxic cyanobacterial blooms - health effects of contact with blooming cyanobacteria, VI, Bioskop, 2006, 1, 13-20 (in Polish).

[18] Edwards D.D., Trouble waters in Milwaukee, Am. Soc. for Microbiology News, 1993, 57 (7), 342 -345 .

[19] Leadership and fecal coliforms: Walkerton 2000, Can. Med. Assoc. J., 2000, 163 (11), 1417.

[20] IwANEJKo R., LubOwIECKA T., Risk analysis in water supply systems. Subject study, Czasopismo Techniczne, 2003, 7, 155-168 (in Polish).

[21] Zімосн I., Integrated method of reliability and safety analysis of water supply systems. Monographs 323, Copyright by Silesian University, Gliwice, 2011 (in Polish).

[22] WIECZYSTY A., Methods of assessing and increasing the reliability of municipal water supply systems, Monographs 2, Environmental Engineering Committee of Polish Academy of Sciences, Kraków, 2001 (in Polish).

[23] JAŹWińSKi J., Borgoń J., Operational reliability and safety of flight, Pub. Communications and Communications, Warszawa, 1989 (in Polish).

[24] POllard S.J.T., Risk Managment for Water and Wastwater Utilities, IWA Publishing, London, 2008.

[25] TCHÓRZEWSKA-CIEŚLAK B., Markov model for risk of failure in water supply system, Polish J. Environ. St., 2009, 2, 74-80. 